\title{
Gestión escolar y desempeño en las instituciones de educación media. Un análisis confirmatorio con sistemas de ecuaciones estructurales
}

\section{School management and performance in secondary education institutions. A confirmatory analysis with systems of structural equations}

\section{Como citar el artículo}

Figueroa, I., (2019). Gestión escolar y desempeño en las instituciones de educación media. Un análisis confirmatorio con sistemas de ecuaciones estructurales. Revista Naturaleza, Sociedad y Ambiente, 6 (1),69-87 DOI: https://doi. org/10.37533/cunsurori.v6i1.42

Idida Maribel Figueroa Alvarez, Amilcar Rolando Corzo Márquez

Universidad Autónoma de Guadalajara, Campus Tabasco, Profesor Titular del Centro Universitario de Petén, USAC

Recibido: 17 de julio de 2019 / Aceptado: 18 de octubre de 2019

Disponible en internet el 29 de noviembre de 2019

*Autor para correspondencia, correo electrónico: idadifigueroa@gmail.com

\begin{abstract}
Resumen
La complejidad de la educación en el nivel medio en Guatemala y la dificultad de reducirla para su entendimiento a formas simples de entender los impactos de organización por medio del conocimiento, el método y análisis de la realidad motivan el interés de este estudio. A pesar de la revalorización social de la función magisterial y la reorganización del sistema lo que implicó la descentralización administrativa, la gestión escolar de las Instituciones Educativas Publicas (IEP) sigue siendo débil. Esencialmente se aborda este problema desde el Desempeño Escolar explicado por la Gestión Escolar por medio del uso de Sistemas de Ecuaciones Estructurales (SEM). Se prueba el impacto negativo de la gestión hacia el Desempeño escolar en el contextos de las (IEP) en el departamento de El Petén, Guatemala. En este estudio se prioriza el problema de investigación fundamentado a través de la teoría de la Gestión Escolar (Pozner, 2000; 1995). El estudio contemplo una muestra de 237 docentes y directores en servicio, las variables del estudio se midieron con un instrumento elaborado y validado para el efecto, y para el desempeño escolar se utilizo datos obtenidos de la aplicación de pruebas estandarizadas de lectura y matemática.
\end{abstract}

Palabras clave: gestión escolar; desempeño escolar; instituciones de educación media

\begin{abstract}
The complexity of education at the middle level in Guatemala and the difficulty of reducing the ability to understand the ways of understanding the effects of the organization in the medium of knowledge, method and analysis of reality motivate the interest of this study. Despite the social revaluation of the teaching profession and the reorganization of the system, which implies administrative decentralization, the school management of the Public Educational Institutions (PEI), remains weak. Essentially this problem is addressed from the School Performance explained by the School Management through the use of Structural Equation Systems (SEM). The negative impact of the management towards the School Performance in the contexts of the (PEI) in the department of Petén, Guatemala is proved. This study addresses the problem of fundamental research through the theory of School Management (Pozner, 2000; 1995). The study contains a sample of 237 teachers and directors in the service, the variables of the study are measured with an instrument developed and validated for the effect, and for school performance are used for standardized reading and math tests.
\end{abstract}

Keywords: school management; school performance; institutions of secondary education 


\section{Introducción}

En la coyuntura moderna se pone al individuo en desventaja en todo momento, iniciando en el medio donde se desenvuelve. En el ámbito educativo se necesita romper paradigmas y llevar a cabo modificaciones acerca de las características del directivo hacia una formación que responda a la sociedad actual. En la perspectiva de la gestión escolar en las instituciones educativas, se han dado cambios en las últimas décadas. Han surgido nuevos enfoques que cambian la mecánica de las escuelas como organizaciones.

Además, conducir una institución educativa implica atender una serie de aspectos referidos a la gestión de la institución y a la gestión de los procesos que ocurren dentro del aula. Cuando se habla de gestionar un centro educativo el director pone en juego sus competencias como un conjunto de saberes y cualidades personales que le permite interactuar con los demás a fin de mejorar el aprendizaje de los alumnos (Le Boterf, 2000, Perrenoud, 1998). Michel Garant (1997:62) afirma que el directivo debe guiar a la organización de manera tal que pueda desempeñar eficazmente su misión atendiendo los objetivos que le dan poder y que a su vez la encuadran socialmente.

Basado en esta tesis, el presente estudio aborda el problema de medir qué tanto la gestión en las IEP impacta en el desempeño escolar de las instituciones educativas, como variable dependiente y efecto inmediato de esta. Esencialmente se resuelve el problema de investigación a partir del uso de los Sistemas de Ecuaciones Estructurales (SEM) como herramienta estadística para la confirmación de hipótesis a partir de la observación científica.
En este sentido se abordó la gestión desde la perspectiva del enfoque estratégico para la construcción de un modelo teórico denominado "gestión escolar» y, coherentemente con este, un modelo estructural en SEM para su confirmación empírica en el contexto 14 IEP con modelos educativos propios y diferentes entre sí, ubicado en los 14 municipios del departamento de Petén, Guatemala, como se describe a continuación.

Revisión de la literatura e hipótesis del estudio

La ciencia de la gestión estratégica de manera general ha derivado en otro grupo de nuevas ciencias como la gestión escolar, que se constituye como el conjunto de procesos y sucesos que se dan al interior de las instituciones educativas. Así la gestión es entendida con el enfoque de la cultura organizacional formada por los docentes y directivos tomando como base las normas, la toma de decisión y los factores y actores que se vinculan de una manera peculiar para realizar las tareas en la escuela, figurando entendimiento entre el logro de los objetivos e identidad como colectivo, (SEP, 2001).

La gestión entonces es un sistema cuyas propiedades o procesos no son reducibles a las de sus partes constituyentes. Al contrario, integra los planteamientos de la administración educativa, pero toma responsabilidad de otras particularidades acordes a la complejidad de las instituciones educativas.

Desde otro punto de vista se percibe a la gestión escolar como el momento que se da dentro de los procesos reales, que dirige a los procedimientos establecidos en las relaciones internas y externas, caracterizada como una acción continua de procesos consistentes con el propósito de orientar la apli- 
cación de los recursos para evidenciar mejor desempeño educativo (Martínez et al 1995). Con todo esto, la gestión escolar además es incluyente de las competencias de los pilares del saber el cual permite constituir y liderar acertadamente a la organización hacia el propósito de la misión regido por un código ético, (Topete 2001).

Asimismo, la gestión escolar emerge de la vinculación que se da entre las personas y los actores de la escuela, (Elizondo et al, 2001). Maquiegui (1997), refiere que en la gestión de una institución escolar, su importancia se percibe cuando se desarrollan las acciones de forma colectiva, con esfuerzo constante previo a establecer acuerdos de los actores de la educación, en ese sentido es vista como un acompañamiento del director hacia los maestros y hacia la comunidad educativa favoreciendo el desarrollo del personal y de la institución.

Sin embargo, los autores Ezpeleta y Furlán, (1992), hablan sobre el enfoque de la gestión pedagógica o procesos sustantivos y añaden que en el contexto organizativo de las instituciones educativas este elemento es esencial y que influye y se articula entre las metas y procedimientos propios del sistema. Otro aporte que hace Topete y Cerecedo (2001) a la gestión escolar, es que perciben que el buen cumplimiento de la misma se determina muchas veces a través de la práctica del poder relacionado puntualmente hacia la forma en la que se toman decisiones al interior de la institución educativa.

Con anterioridad a los aportes de los autores mencionados Namo de Mello (1998) y Guadamuz (1998), ya habían manifestado que la gestión escolar se conforma con todas las acciones que se realizan en el contexto to- mando en cuenta las diferentes dimensiones desde el centro escolar, de tal manera que la institución logre retribuir los intereses y necesidades de los escolares. De acuerdo a los aportes de los autores en mención, hacia la gestión escolar han prevalecido tres perspectivas: primeramente figuran las influyentes en la parte administrativa con énfasis en lo organizacional (Martínez et al; 1995 Navarro, 1999; Topete y Cerecedo 2001), mientras que en la segunda resalta idea de los procesos, la cotidianeidad, los sujetos y la cultura de lo escolar (Ezpeleta y Furlán, 1992, Pastrana, 1997; Elizondo et al, 2001; Marquiegui, 1997) en último término la tendencia que resalta el fin concluyente de la gestión escolar: la generación de aprendizajes en la escuela (Schiefelbein, 1997; Namo, 1998; Guadamuz; 1998).

No obstante la tarea de la gestión educativa no es simple ni se le atribuye a un solo factor, es compleja y puede entenderse como una nueva forma de interpretar la organización escolar, considerando la mediación entre el futuro y el presente, prever aunque la predicción no sea posible, mantener una reacción rápida y planeada ante las circunstancias, mediando el conocimiento y la acción para ello se necesita una acción centrada coherente integral contrariamente a las acciones parciales de los querellantes sociales, (Pozner 2000).

De acuerdo con Loera (2004), la gestión escolar es entendida como un cúmulo de acciones que realiza la comunidad educativa (director, maestros, personal de apoyo, padres de familia y alumnos), orientadas con la labor fundamental que le ha sido atribuida a las instituciones escolares.

La gestión escolar es estratégica cuando logra que las competencias y las experiencias se vinculen. $Y$ tiene que ver con designar la eficacia, calidad y buena orientación de 
la intervención en las escuelas, el equilibrio entre lo técnico y lo político referido a la educación; únicamente a través de la revisión del proceso estratégico de las prácticas de dirección de las organizaciones educativas puede hablarse de gestión (ILPE-UNESCO: 2000).

\section{Dimensiones de la Gestión escolar}

Tal como se ha registrado la evolución de la gestión escolar en la literatura, su desplazamiento ha tenido cambios en los enfoques de la gestión, en primera instancia se vieron sujetos a revisar la capacidad que tendrían las escuelas para superar las desigualdades de origen de sus alumnos, pero no mostro mayor relevancia se mantuvo en un plano de forma subordinada (Coleman 1969; y Jencks 1972), sin embargo más adelante los procesos de la gestión cobran el sentido de identificar factores de incidencia escolar (Creemers 1994; Slavin 1994; Schereens 1992), similares conclusiones se encuentran en diversas investigaciones internacionales. Así, Arancibia (1992), Espinoza (1995), Zárate (1992), Servat, (1996) y Alvariño et al (1999). Seguidos de estudios que afirmaban la existencia y tratamiento de las variables relevantes de gestión (Gardner 1995 y Bruner 1996; The World Bank 1998;), (Murnane y Levy 1996).

Muy recientemente la literatura registra que los enfoques de esta teoría se han revisado y se han centrado propiamente en la gestión escolar, distinguiendo innovaciones relacionadas con procesos de descentralización entre ellos: la gestión basada en la escuela su responsabilidad y relación entre escuela y comunidad (OECD 1992, McDonell 1989; IIPE 1999, Gómez 1998; Revista Iberoamericana de Educación 1993, 1994; Lesourne 1993).
De acuerdo a diversos estudios que se han realizado sobre el tema de gestión en las instituciones educativas, es necesario desarrollar dimensiones que enmarquen el cumplimiento de las actividades que realizan dichas instituciones de igual manera tener los objetivos claros para lograr la misión y visión de los establecimientos. En ese escenario de la realidad escolar, es que se hace necesario clasificarla en dimensiones. Desde la perspectiva analítica, que permite ser una herramienta que sirva para observar, analizar, realizar crítica constructiva e interpretar lo que sucede a lo interno de la organización y su quehacer diario. Ante todo, las dimensiones de la gestión escolar son el ámbito donde se percibe el proceder y toman fuerza y significado las actuaciones de los actores de la educación, considerándolos como medios donde se observa la actuación activa e interactiva instantánea de la realidad educativa vista desde la parte institucional, escolar y lo pedagógico.

Para efectos del presente estudio las dimensiones que se plantearon son cinco: Procesos sustantivos, (pedagógico curricular) Organización del trabajo, Administración del recurso, Apoyo a estudiantes y Gestión, (Pozner 1995; Braslavsky, 1992; Mendoza, 2011).

\section{Procesos Sustantivos}

A nivel de instituciones educativas la gestión se lleva a cabo por medio de diversos procesos y actuaciones indudablemente el proceso pedagógico curricular constituye el elemento primordial del ser de las escuelas. No obstante esta dimensión no es independiente de las demás, dichos procesos no están al margen de otras categorías organizativas, pero en la medida que se desarrolla la actividad educativa involucra la participación de alguna manera de las demás dimensiones. 
Los procesos sustantivos aparecen adjetivados como la dimensión pedagógica curricular. Representa los fines y objetivos específicos o razón de ser de la institución en la sociedad. Contiene las prácticas particulares de la enseñanza y aprendizaje, los contenidos, textos y auxiliares didácticos, las prácticas de evaluación, entre otras (Pozner, 1995). Estos analizan las formas de enseñanza y los factores asociados a éstas en la aplicación de los enfoques curriculares. Según la Secretaria de Educación Pública, (SEP, 2006), son procesos sustantivos y fundamentales del quehacer de la escuela y sus actores, incluyen: la enseñanza y el aprendizaje y otros factores como, planeación, evaluación, clima de aula, uso del tiempo destinado a la enseñanza y recursos de apoyo (Frigerio, Poggi, Tiramonti y Aguerrondo, 1992).

Por otro lado, es importante reconocer los niveles de logro educativo que presentan los alumnos de la escuela para iniciar un proceso de mejora. El currículo Nacional Base Guatemalteco (CNB 2009) enmarca que la metodología que el docente debe ocupar para el logro de las competencias en los escolares, propiciando oportunidades de desempeño para aplicación en su entorno inmediato.

Finalmente es evidentemente que el componente principal es la tarea del maestro, el cual determina, el logro o no del estudiante. Reconociéndola como una de las funciones esenciales (SEP, 2009) para que los alumnos logren un desempeño polifuncional en diversas situaciones $y$, sobre todo, enriquezcan la perspectiva de sí mismos, como ciudadanos y como seres humanos sensibles e inteligentes.

\section{Organización del trabajo}

Esta dimensión hace referencia a cómo está organizado el centro escolar, como sus elementos se articulan de manera formal es la base donde se sostienen las demás, esta dimensión es reconocida como estructural. Santos (1997, 1999), añade que la institución educativa es una organización formal por contener roles de los actores educativos de acuerdo con su estructura, el mismo permite darle estabilidad y continuidad en el tiempo y le permite actuar tomando en cuenta las características personales de sus integrantes. Dentro de esta dimensión se percibe elementos formales establecidos que forman el andamiaje en el que se lleva a cabo la labor educativa.

Sus elementos juegan papeles y roles correspondiente a las tareas y responsabilidades. En ese sentido también se refleja las estructuras de tareas, profesor alumno, horarios, regulación de la utilización de la infraestructura. Cabe mencionar que en la medida que las estructuras de las instituciones escolares están regidas por las normativas y fundamentos legales emanados de administración educativa (Santos Guerra, 1997; Beltrán y San Martín, 2000) o sea que forman parte de un mismo sistema educativo, poseen una estructura formal similar.

Pozner (1995) indica que la forma organizativa de la gestión escolar se representa a través del órgano interno de la institución educativa entendiendo los subsistemas, que los conforman. Así esta dimensión logra la organización de los actores de la educación, aprovechamiento racional de los recursos, toma de decisiones oportunas, y relación entre la comunidad educativa de centro escolar. Además llevan a cabo periódicamente la evaluación de los avances que han tenido, 
se autoevalúan como una manera de mejorar sus indicadores de logro, ILPE (2003).

\section{Administración del recurso}

La comprensión de esta dimensión admite que, desde la administración escolar, se puede dar apoyo a los procesos educativos planeados con el objetivo de hacerles cambios que permitan mejorarlos enfatizando en el rendimiento de los escolares, el desenvolvimiento tanto de los docentes como del director y personal de apoyo. De esta manera la dimensión administrativa financiera está referida a la distribución del tiempo y del espacio del establecimiento; así como la administración de los recursos humanos, materiales y financieros de la institución escolar (Pozner 1995).

La SEP (2009), en el Modelo de Gestión Educativa Estratégica, (MGEE) indica que el propósito de esta dimensión es valorar los objetivos y actividades que se desarrollan en las reuniones de Consejo Técnico, así como el liderazgo directivo que se ejerce para la consecución de las mismas, el seguimiento a los acuerdos y compromisos y su trascendencia en el clima organizacional prevaleciente de la escuela.

\section{Apoyo a estudiantes}

Según Mendoza (2011) es un aspecto crucial al interior de las salas de clase es la capacidad del profesor para generar interés por el aprendizaje en sus alumnos. Esto se relaciona estrechamente con su disposición a comprometerse con los contenidos que enseña y con lograr que sus alumnos se motiven y valoren lo que están aprendiendo. Estas salas de clases se distinguen porque son ambientes activos, en los cuales, tanto el profesor como los estudiantes se formulan preguntas, están interesados en indagar y llegar a resultados y productos satisfactorios y en comunicar a otros sus hallazgos. En estos ambientes los alumnos se sienten desafiados a aprender y son apoyados por el profesor, quien cree realmente que todos sus alumnos y alumnas pueden aprender y esforzarse para ello, favoreciendo el ejercicio de su autonomía.

De esta manera, los estudiantes no temen al ridículo cuando proponen ideas, preguntas 0 temas de su interés, ya que saben que ese es un espacio para aprender y cuestionarse, y que el profesor se interesará por sus aportes. Los alumnos se sienten seguros porque el profesor refuerza su compromiso con lograr buenos resultados y trabajos bien hechos y no enfatiza la sola realización de la actividad - la tarea, sino que la calidad de la misma, ofreciendo ayudas para que ésta se alcance progresivamente. En tal sentido, es de gran importancia que el profesor genere y transmita a sus alumnos altas expectativas de manera que superen las situaciones adversas (MINEDUC -Chile 2005).

\section{Gestión curricular directiva}

El compromiso del docente con la labor que desempeña comprende no sólo su trabajo en el aula, sino también las relaciones que establece con sus colegas. El profesor/director se considera y actúa como miembro de una comunidad de aprendizaje con la cual comparte responsabilidades que se extienden más allá de su aula. La reflexión colectiva y el trabajo en equipo con el resto de los profesionales del establecimiento constituye un elemento fundamental de la labor docente que le permite mejorar sus prácticas, mejorar el conocimiento de sus alumnos, así como sus pro- 
pios conocimientos. Para MINEDUC (2005), los equipos directivos trabajan permanentemente para comprender, mejorar y potenciar las capacidades, las habilidades personales y la motivación de los docentes y asistentes de la educación de su establecimiento, así como su propio desarrollo profesional, a manera de construir capacidades internas que permitan alcanzar los objetivos y metas de mejoramiento del establecimiento y sostenerlas en el tiempo.

Además, implementan estrategias de inducción para los nuevos docentes de manera que se apropien de las normas de funcionamiento y de la cultura del establecimiento. Por otro lado, desarrollan estrategias de retención para mantener un cuerpo docente estable, motivado y comprometido, mediante el mejoramiento de sus condiciones laborales y la generación de un clima de confianza y desafiante profesionalmente, todo lo cual ayuda fuertemente en la sostenibilidad de los procesos de mejoramiento del establecimiento (Mendoza 2011).

\section{Desempeño en la gestión escolar}

Con relación a la eficacia escolar se han desarrollado una abundante literatura en menos de un siglo de existencia, en el mismo se evidencia la contribución a la mejora de la educación en general. La eficacia escolar se considera en otros términos como el desempeño de la institución escolar, ha demostrado un escenario de contextos diversos y características que abarcan cada una de sus etapas a la organización escolar. La comprensión y aportes teóricos de la investigación educativa en relación a la eficacia es una ventana que ha permitido vincular visiblemente con una de las variables de proceso de mayor respaldo en la contribución a la explicación de diferentes productos educativos, entre ellos: logros académicos, prestigio, innovación, a nivel de centros docentes como es el liderazgo activo de los directivos.

En ese sentido existen diversos estudios que permiten evidenciar aquellos aspectos propios del proceso de enseñanza y del docente que influyen en la consecución de logros del estudiante. Taylor (1962) define la eficacia escolar como (proceso-producto), es el primero que trató de identificar las características percibidas de un buen docente. Mientras que Weber (1971), realizó un estudio donde seleccionó escuelas con estudiantes de altos rendimientos lectores y cuya mayoría perteneciese a clase socioeconómica baja. También en un estudio internacional realizado por Murillo (2007), trata de describir los efectos escolares, de aula y de país en la educación primaria en Iberoamérica y encontrar los factores de eficacia escolar. Para estimar la eficacia de la escuela se deben acompañar las altas puntuaciones en el rendimiento de los estudiantes verificando también el desarrollo de aspectos socioemocionales. Este estudio es considerado como el más importante llevado a cabo por la UNESCO en Iberoamérica que da pie a profundizar cuáles son los factores consensuados por los investigadores en la investigación en eficacia escolar en Iberoamérica.

En resumen es evidente que la investigación en eficacia escolar ha sido capaz de ofrecer hallazgos muy significativos para la toma de decisiones en las políticas educativas y para la propuesta de programas de mejora en los centros. A través del recorrido de las investigaciones referenciadas se percibe la evolución del Movimiento de Eficacia Escolar. Todos estos avances, permiten ahondar en el estudio de la Educación bajo un interés común: 
alcanzar el desarrollo integral de todos los/las estudiantes considerando las condiciones de partida propias, el apoyo que la investigación en Eficacia Escolar aporta a los sistemas educativos actuales contribuye a reconducir las escuelas hacia la igualdad de oportunidades, maximizar el tiempo en ambientes educativos.

\section{Indicadores de Desempeño de Institucio- nes Educativas}

Dentro de los estudios de efectos escolares se ha evidenciado que existen herramientas objetivas que permiten darle un valor a las propuestas educativas nacionales, ya sea como producto de los procesos de evaluación o mejora institucional o como parte de las políticas educativas. Según Martínez (2009), su uso ha sido reconocido en procesos de rendición de cuentas y mejora escolar.

Dentro de los estudios realizados en América Latina, y que han cobrado mayor interés en cuyos objetivos estaba estimar la magnitud de los efectos escolares; dos de los estudios regionales fueron desarrollados por el Laboratorio Latinoamericano de Evaluación de la Calidad de la Educación, LLECE, OREALC/ UNESCO, y uno coordinado por Murillo $y$ Torrecilla y denominado Investigación Iberoamericana sobre Eficacia Escolar (Murillo-Torrecilla, 2007).

Los tres emplean como instrumentos de medición pruebas estandarizadas, para las áreas, y cuestionarios de factores asociados que diligencian los estudiantes, la familia, el docente, y el director-escuela. El primero encontró que los efectos para la región son de $15,5 \%$ en Lengua y $18,3 \%$ en Matemáticas (LLECE, 2006).El segundo trabajo desarrollado señalan que, en contraste con los estudios anglosajones, el aporte de la escuela para el conjunto de América Latina y el Caribe es superior al $30 \%$ en las áreas evaluadas, porcentaje que se considera alto para el conjunto del continente (OREALC/UNESCO, 2010). El tercer estudio, la Investigación Iberoamericana sobre Eficacia Escolar (Murillo-Torrecilla, 2007) que, aunque analizó menos países. Sus resultados señalan que el porcentaje en que la escuela influye en el logro académico cognitivo se encuentra entre $14 \%$ y $18 \%$, estando cerca del $10 \%$ cuando se descuenta la influencia del aula. El porcentaje es mayor para matemáticas que para Lengua.

En torno al tema de un sistema de Indicadores Educativos en Guatemala se han dado una serie de propuestas que permiten en diferentes niveles dar un seguimiento a los indicadores tradicionales. Las mismas han partido de las necesidades coyunturales o desde las políticas vigentes dentro del Ministerio de Educación para responder a necesidades puntuales. Tanto es así que en el año 2009, se propusieron una serie de Indicadores como parte de un esfuerzo del Ministerio de Educación, USAID, Diálogo para la Inversión Social en Guatemala y AED.

Seguidamente en el año 2010, se utilizaron una serie de indicadores que respondieron en su momento a las necesidades de información y de reporte de información del MINEDUC, por medio del SNIE el cual se considera un mecanismo mediante el cual se puede evaluar objetivamente, de forma interna o externa, la evolución del sistema educativo y el cumplimiento del mandato constitucional.

A partir del 2011, se replantea los indicadores con el objetivo de responda a las demandas de información y a las necesidades de monitoreo del sistema educativo, así como que le den seguimiento a las políticas educativas 
actuales.

Finalmente en el año 2012 se estableció un sistema de indicadores educativos -SNIE-, que incluyeron nuevos indicadores que permitan medir y comparar internacionalmente la situación de la educación en Guatemala, así como la capacidad del sistema educativo nacional. El SNIE, está compuesto por indicadores divididos en 5 dimensiones que constituiría la oportunidad de establecer una línea de base comparable en el tiempo, que incluye: a. La definición de indicadores, b. La construcción o cálculo de los mismos, y c. La actualización y la mejora continua del propio sistema de indicadores.

El modelo de las dimensiones fue una adaptación del modelo de Calidad Educativa propuesto por la UNESCO en el año 2005, que están constituidas por:

a. Contexto de la educación.

b. Recursos. c. Procesos educativos.

d. Resultados de escolarización y eficiencia interna.

e. Resultados de aprendizaje del sistema.

El presente estudio tomó únicamente las dimensiones que hacen referencia a los resultados de escolarización y eficiencia interna, los cuales agrupan una serie de variables que permiten caracterizar el acceso, ingreso y el transcurrir de los estudiantes por el sistema educativo, el tiempo que les toma a los estudiantes finalizar un nivel educativo determinado, la cobertura para los distintos niveles del sistema y segundo lugar los resultados de aprendizaje del sistema estos agrupan los resultados de las pruebas estandarizadas en las áreas de Lectura y Matemáticas, que miden parte de lo que los estudiantes aprenden a su paso por la escuela.

\begin{tabular}{ll}
\hline Indicadores & Dimensiones \\
\hline Estudiantes inscritos que no desertan & Tasa de retención \\
Estudiantes que aprueban & Tasa de Aprobación \\
Estudiantes de cohortes que se gradúan & Eficiencia Terminal \\
Desempeño de Matemática y Lenguaje & Resultado Pruebas Nacionales \\
\hline
\end{tabular}

Tabla 1. Indicadores y Dimensiones de desempeño escolar.

Nota: Elaborado en base SNIE Guatemala.

En resumen y de acuerdo a la literatura consultada, la gestión escolar debe enfocarse hacia una nueva forma de interpretar la organización escolar, considerando la mediación entre el futuro y el presente, mantener una reacción rápida y planeada ante las circunstancias, mediando el conocimiento y la acción para ello se necesita una acción cen- trada coherente integral contrariamente a las acciones parciales de los querellantes sociales, (Pozner 2000), y que estas acciones influyen en el desempeño escolar de los estudiantes. Derivado de lo anterior, se plantea la hipótesis del presente estudio: La Gestión Escolar impacta en el Desempeño Escolar de las Instituciones educativas. 


\section{Metodología}

La investigación es de corte cuantitativo, sigue una lógica deductiva y utiliza los SEM como método estadístico para probar su hipótesis. Su alcance es correlacional, a partir de un estudio transversal en el tiempo de las variables relevantes, y confirmatorio. En este sentido se obtuvieron los datos relativos a los atributos observables en el trabajo individual del personal de las instituciones educativas, como variables medibles. Se midieron los atributos a nivel individual, al abordar a docentes y administrativos con más de un año de laborar en la institución, aunque no fueran de tiempo completo. La intervención se realizó a través de una encuesta autoadministrada. La intervención se limitó a los institutos de educación media en un mismo período lectivo.

\section{Instrumentos de medición}

Se diseñó un cuestionario dada la naturaleza y objetivos de esta investigación, se requirió del diseño y validación de un cuestionario para medir la percepción que tiene los docentes del nivel medio en relación la Gestión Escolar en las IES. Debido a la falta de un instrumento que mida las variables latentes del constructo de Gestión Escolar se revisó la literatura cualitativa que existe a cerca del constructo y se seleccionaron los factores luego se elaboraron los itemes que la contenían. Se hace una revisión de la teoría de la gestión estratégica para determinar el contenido unidimensional, posteriormente se realiza la definición y ordenación, finalmente se llega al proceso de validación y la prueba piloto aplicada a 60 personas

Para analizar la consistencia interna del cuestionario, se llevó a cabo la prueba piloto. Tanto en esta etapa como en la siguiente, los ítems del instrumento aparecieron agrupados en sus respectivas dimensiones, a fin de poder realizar, con posterioridad, los análisis estadísticos que permitieran definir su adecuación en cada una de las dimensiones. En un primer análisis, se obtuvo un coeficiente de Alfa de Cronbach de 0,968, el cual excede el valor mínimo de 0,70 para determinar la confiabilidad de la prueba (DeVellis, 2003; Kline, 2000).

Para la aplicación del instrumento se elaboró un plan de ejecución dirigido a los establecimientos educativos sujetos de estudio, posteriormente se coordinó con los directivos de cada centro para informar el propósito de investigación y consensuar fechas de intervención de recogida de datos a los sujetos seleccionados. Los datos obtenidos a través de la aplicación del cuestionario fueron analizados con el programa estadístico SPSS 22.0. Se garantizó estadísticamente su validez y confiabilidad.

\section{Pruebas prácticas de lectura y matemática para medir desempeño escolar}

Para ello se obtuvo las pruebas liberadas de matemática y lectura que aplica el Ministerio de Educación a través de Dirección General de Evaluación Educativa (DIGEDUCA) a estudiantes del tercer grado básico de las instituciones sujetas de estudio. En la primera parte de la prueba recoge datos del estudiante, en la segunda contiene 25 enunciados así como las cuatro posibles respuestas u opciones que la completan. Los resultados de ambas pruebas se analizaron con teoría clásica.

La muestra contemplo una muestra de 237 docentes y directores en servicio de instituciones educativas en 14 municipios del departamento de Petén en Guatemala. 


\section{Resultados}

\section{Análisis descriptivos de la Variables}

En este apartado se describen la distribución de respuestas de las dimensiones de GE. EI instrumento que mide los valores de GE se compone de 49 ítems en su totalidad formada por cinco dimensiones y desempeño escolar con indicadores de permanencia y resultados de pruebas estandarizadas de lectura y matemática.
Para reportar el análisis de validez discriminante y convergente de las variables latentes y con ello probar si las variables de un constructo de medida son conceptualmente diferentes o si deben constituirse en una sola variable. Se estableció el Alfa de Cronbach, la fiabilidad compuesta y la validez convergente considerando los ítems de cada dimensión del análisis factorial confirmatorio, ver Tabla 2.

\begin{tabular}{lccccc}
\hline \multicolumn{1}{c}{ Constructo } & $\begin{array}{c}\text { Alfa de } \\
\text { Crombach }\end{array}$ & $\begin{array}{c}\text { Fiabilidad } \\
\text { compuesta } \\
\text { (pc) }\end{array}$ & Interpretación (pc) & $\begin{array}{c}\text { Validez } \\
\text { compuesta (AVE) }\end{array}$ & $\begin{array}{c}\text { Interpretación } \\
\text { (AVE) }\end{array}$ \\
\hline $\begin{array}{l}\text { Procesos Sustantivos } \\
\text { Apoyo a estudiantes }\end{array}$ & 0.842 & .9559750230 & Fiabilidad superior a 0.7 aceptable & 0.685 & Aceptable \\
$\begin{array}{l}\text { Gestión Curricular } \\
\text { Directiva }\end{array}$ & 0.873 & .9694695851 & Fiabilidad superior a 0.7 aceptable & 0.743 & Aceptable \\
$\begin{array}{l}\text { Organización del } \\
\text { Trabajo }\end{array}$ & 0.951 & .9825280024 & Fiabilidad superior a 0.7 aceptable & 0.889 & Aceptable \\
$\begin{array}{l}\text { Administración del } \\
\text { Recurso }\end{array}$ & 0.882 & .9688917083 & Fiabilidad superior a 0.7 aceptable & 0.757 & Aceptable \\
\hline
\end{tabular}

Tabla 2.Validez y análisis de los Constructos de Gestión Escolar.

Elaboración propia con base al estudio.

La tabla 2 ilustra la fiabilidad compuesta en cada uno de los valores y sus coeficientes son $(0.955,0.969,0.982,0.968,0.966$,$) res-$ pectivamente lo cual se ubican en la categoría de suficiente porque superan el criterio empírico 0.70 sugerido por Nunally y Bernstein (1974) cumple con el factor de la validez convergente y fiabilidad.

De igual manera se determinó la Varianza Extraída Promedio (AVE), para cada factor (ver tabla 2) en todos los factores la varianza es superior a (0.5) (criterio Hair 1995), y que la fiabilidad compuesta es (0.846) permitió concluir en el análisis factorial confirmatorio.

\section{Análisis factorial}

El análisis factorial extrajo para Gestión Escolar cinco componentes principales, cargas aceptables por encima de 0.70 , una varianza explicada de $53.5 \%$ de los valores originales medida de adecuación muestral de Kaiser-Meyer-Olkin de 0.928 cumple con el criterio, significancia de prueba de esfericidad de Bartlett con valor de 0.000 que es menor a 0.05 (significativo si $P<0,05$ ) lo que confirma la conveniencia del análisis factorial e indica su pertinencia por existencia de correlaciones significativas entre variables. En virtud del análisis de fiabilidad se demuestra que la dimensión Gestión Escolar no es unidimensional e integra medición óptima de las variables que la conforman. 


\begin{tabular}{lc}
\hline & Estadística de fiabilidad \\
\hline & Alfa de Cronbach \\
Procesos sustantivos & 0.842 \\
Apoyo a Estudiantes & 0.873 \\
Gestión curricular directiva & 0.951 \\
Organización del trabajo & 0.882 \\
Administración del recurso & 0.863 \\
\hline
\end{tabular}

Tabla 3. Análisis de fiabilidad para la escala Gestión Escolar.

Elaboración propia con base en resultados.

Los resultados para el cálculo del coeficiente alfa de Cronbach se tendría calculando la varianza de los ítems del factor Gestión Escolar el valor más bajo que se encontraría en 0.842 la dimensión que reporta el valor más bajo que se encontraría dentro del límite de 0.7 a 0.9 que indica una buena consistencia interna para esta escala (ver tabla 3).

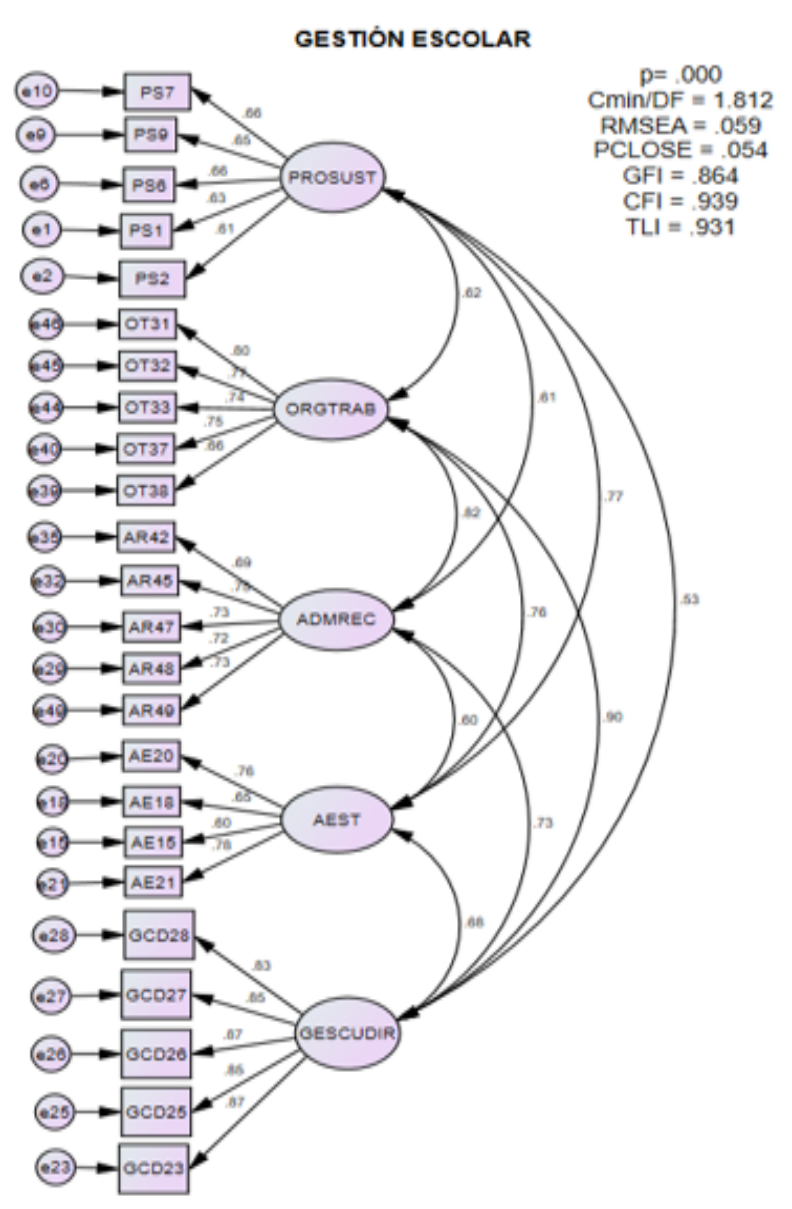

Figura 1.Modelo de Gestión Escolar.

Nota: Elaboración propia para el presente estudio.

\section{Análisis Confirmatorio}

Para llevar a cabo el análisis factorial confirmatorio se empleó el paquete AMOS 22 con el propósito de disminuir el número de ítems que conforman los instrumentos, de tal manera que el mismo tenga características manejables y de parsimonia y de esta manera probar el ajuste ideal del modelo en la muestra de estudio. Para ello se tomaron referencias de Uriel y Aldás (2005). El principio de este análisis se recoge con la dimensión Gestión Escolar el instrumento fue construido en base a la perspectiva teórica de la gestión estratégica, como se observa en la Figura 1 está integrado por 5 dimensiones y 24 variables.

En relación a la dimensión Gestión escolar el ajuste de los datos se integró con 24 ítems siendo estos: Procesos sustantivos con $1,2,6,7$ y 9 , Apoyo Escolar con $15,18,20$ y 21 ; Gestión Curricular Directiva con 23,25,26,27 y 28; Organización del Trabajo con 31,32,33,37 y 38; mientras que Administración de Recurso se integró con los ítems 42,45, 47,48 y 49. Ver figura 1. 


\begin{tabular}{|c|c|c|}
\hline $\begin{array}{c}\text { Condición } \\
\text { de ajuste }\end{array}$ & $\begin{array}{c}\text { Considerando } 24 \\
\text { ítems }\end{array}$ & Criterios que debe cumplir \\
\hline$\chi^{2}$ & 0.54 & $\mathrm{p}\left(\chi^{2}\right)$ debe ser superior a 0.05 \\
\hline $\mathrm{X}^{2 / g l}$ & 1.812 & Debe ser inferior a 3 \\
\hline GFI & 0.864 & Debe ser mayor a 0.95 \\
\hline RMSEA & 0.59 & Admisible un valor inferior a 0.0 .8 \\
\hline CFI & 0.939 & Debe ser mayor a 0.95 \\
\hline
\end{tabular}

La figura 1 muestra los resultados del análisis factorial confirmatorio (AFC). La gráfica muestra los resultados del AFC utilizando los ítems con escala ordinal directamente de la base datos y el método de mínimos cuadrados generalizados. Presentando una probabilidad mayor que $(p>0.05)$ y una relación $\mathrm{X} 2$ y grados de libertad menor a dos (Cmin/ $\mathrm{DF}=1.812$ ) es un indicador de muy buen ajuste estadístico de los datos al modelo, que además se confirma con los valores de los indicadores RMSEA $=0.59$ y PCLOSE $=0.054$, GFI $=0.864$, aunque lo esperado es arriba de 0.95 es un dato aceptable para el AFC, y $\mathrm{CFI}=0.939$ y todos los parámetros estimados apropiados.

Seguidamente se explican los resultados del análisis confirmatorio realizado como parte del modelo conjunto de estudio. Los resultados presentados verifican ajuste del modelo propuesto de relación causal entre las dimensiones. En la Tabla 4 se exponen los resultados del análisis factorial confirmatorio obtenido encontrando cargas factoriales de las variables calculadas por encima de 0.077 . Las fiabilidades compuestas tienen valores por encima de 0.077 y las varianzas medias extraídas oscilan entre 0.411 y 0.728 . Los coeficientes de correlación múltiple del modelo ajustado reflejan valores de asociación entre las variables Procesos Sustantivos (PS) Apoyo a Estudiantes (AE) Gestión Curricular Directiva (GCD) Organización del trabajo (OT) Administración de Recurso (AR) con valores que explican su varianza de 0.641 , $0.746,0.724,0.702$ y 0.853 respectivamente.

Tabla 4

Analis is factorial confirmatorio del modelo global de Gestión Escolar

\begin{tabular}{lcccccccc}
\hline CONSTRUCTO & FC & AVE & MSV & PROSUST & ORGTRAB ADMREC & AEST & GESCUDIR \\
\hline PROSUST & 0.777 & 0.411 & 0.598 & 0.641 & & & & \\
ORGTRAB & 0.862 & 0.557 & 0.815 & 0.623 & 0.746 & & & \\
ADMREC & 0.847 & 0.525 & 0.669 & 0.614 & 0.818 & 0.724 & & \\
AEST & 0.793 & 0.500 & 0.598 & 0.773 & 0.757 & 0.599 & 0.702 & \\
GESCUDIR & 0.930 & 0.728 & 0.815 & 0.529 & 0.903 & 0.733 & 0.678 & 0.853 \\
\hline
\end{tabular}

Fuente: Elaboración propia y resultados del modelo en sofware AMOS 
En la tabla 5 contiene las estimaciones de los parámetros del modelo, el error estándar aproximado, la proporción crítica y las estimaciones estandarizadas de los parámetros. La proporción crítica es el cociente entre la estimación del parámetro y la estimación del error estándar. Si se reúnen las suposiciones de distribución apropiadas, el estadístico sigue una distribución normal estándar bajo la hipótesis nula de que el parámetro tiene un valor de cero. Como se puede observar en la tabla todos los valores de proporción crítica son grandes, obteniendo de esta forma que todos los parámetros estimados son significativos.

El modelo de relación de ecuaciones estructurales permite analizar el comportamiento de cada una de las variables contra las demás, como se observa en la Figura 5, se tienen los valores estandarizados de cada factor, por medio del paquete computacional AMOS, de la hipótesis perteneciente a esta investigación.

Tabla 5

\begin{tabular}{|c|c|c|c|c|c|c|}
\hline & & Estimate & S.E & C.R & P Label & \\
\hline PS1 & $<---$ PROSUST & 0.882 & 0.11 & 8.037 & $* * *$ & \\
\hline PS6 & $<---$ PROSUST & 0.904 & 0.109 & 8.294 & $* * *$ & \\
\hline PS9 & $<---$ PROSUST & 1.078 & 0.132 & 8.157 & $* * *$ & \\
\hline AE15 & $<---$ AEST & 0.652 & 0.075 & 8.716 & $* * *$ & \\
\hline AE18 & $<---$ AEST & 0.83 & 0.087 & 9.577 & $* * *$ & \\
\hline AE20 & $<---$ AEST & 1 & & & $* * *$ & \\
\hline AE21 & $<---$ AEST & 1.104 & 0.96 & 11.451 & $* * *$ & $\nabla$ \\
\hline GCD23 & $<---$ GESCUDIR & 1 & & & $* * *$ & GESTESC \\
\hline GCD25 & $<---$ GESCUDIR & 1.005 & 0.058 & 17.404 & $* * *$ & \\
\hline GCD26 & $<---$ GESCUDIR & 0.926 & 0.051 & 18.163 & $* * *$ & 1 de Estudio Gestión Escolar, Desempeño Escolar. (Variables medir \\
\hline GCD27 & $<---$ GESCUDIR & 0.951 & 0.054 & 17.601 & $* * *$ & \\
\hline AR48 & $<---$ ADMREC & 1 & & & $* * *$ & \\
\hline AR47 & $<---$ ADMREC & 0.796 & 0.076 & 10.469 & $* * *$ & \\
\hline AR45 & $<---$ ADMREC & 0.983 & 0.091 & 10.788 & $* * *$ & \\
\hline AR42 & <--- ADMREC & 1 & 0.1 & 9.98 & $* * *$ & \\
\hline OT38 & <--- ORGTRAB & 1 & & & $* * *$ & \\
\hline OT37 & $<---$ ORGTRAB & 1.148 & 0.113 & 10.2 & $* * *$ & \\
\hline OT33 & <--- ORGTRAB & 1.491 & 0.148 & 10.043 & $* * *$ & \\
\hline OT32 & $<---$ ORGTRAB & 1.337 & 0.128 & 10.415 & $* * *$ & \\
\hline OT31 & $<---$ ORGTRAB & 1.434 & 0.134 & 10.72 & $* * *$ & \\
\hline AR49 & $<---$ ADMREC & 0.921 & 0.088 & 10.453 & $* * *$ & \\
\hline PS7 & $<---$ PROSUST & 1 & & & $* * *$ & \\
\hline GCD28 & $<---$ GESCUDIR & 0.852 & 0.051 & 16.683 & $* * *$ & \\
\hline PS2 & $<---$ PROSUST & 0.888 & 0.114 & 7.795 & $* * *$ & \\
\hline
\end{tabular}

Nota: Software Versión 20

De acuerdo a los resultados de este modelo general se presenta la comprobación de la hipótesis con los valores del análisis de regresión, de acuerdo los datos obtenidos. 
H1.Existe una relación positiva entre Gestión Escolar y Desempeño Escolar. Esto se comprueba mediante el modelo general, el peso de regresión estandarizado de gestión escolar y desempeño escolar de $\lambda=-0.021, \mathrm{p} .=$ 0.788 se rechaza la hipótesis.

\section{Discusión}

En este apartado se presenta la discusión entre la teoría revisada en la investigación, la cual ha sido mostrada en la revisión de la literatura, y los resultados obtenidos en el trabajo de campo a través de los instrumentos aplicados a docentes y directores de las instituciones educativas.

Con fundamento en la literatura consultada, se consideró que el constructo Gestión Escolar se integra por las dimensiones Procesos Sustantivos, Apoyo a Estudiantes, Gestión Curricular Directiva, Organización del Trabajo, Administración de Recurso (Pozner 1995).

El constructo Gestión Escolar se midió a través de un cuestionario elaborado y validado modelado por medio de la teoría de la Gestión Estratégica con el aporte de Pozner (1995), Mendoza (2011) compuesta por cinco dimensiones, 24 ítems y una escala Likert de cinco respuestas, se confirma que la Gestión se compone de las cinco dimensiones mencionadas antes.

El análisis de resultados se realizó mediante comparación de la información obtenida con las hipótesis estadísticas de investigación. La Gestión Escolar del modelo de investigación se integra por los ítems PS con 1,2,6,7 y 9 , AE con $15,18,20$ y 21 ; GCD con $23,25,26,27$ y 28 ; OT con $31,32,33,37$ y 38 ; mientras que AR se integró con los ítems 42,45, 47,48 y 49. Las medias de respuesta son superiores a 3.61 de una escala Likert de uno a cinco en todos los casos. Los resultados obtenidos en el modelo SEM reflejan que los valores estandarizados oscilan entre 0.66 y 0.80 .

En el planteamiento del estudio se explicó que la gestión escolar enfatiza en las dimensiones que son indispensables para llevar a cabo los procesos organizacionales en las instituciones educativas.

Para el enfoque de gestión escolar, y se partió de la teoría de la gestión estratégica de Pozner (1995), Cecilia Braslavsky (1992, Mendoza (2011) Alvariño (2000, OECD (1992), McDonell (1989, el cuestionario utilizado para medir el constructo, se construyó y valido debido que no se encontraron estudios relacionados que permitieran medir las diferentes dimensiones que la teoría enfatiza para garantizar la gestión escolar. El modelo teórico confirmado permite responder la pregunta de la investigación ¿De qué manera la Gestión Escolar impacta en el Desempeño Escolar? La conclusión del estudio resuelve que "La gestión escolar se relaciona negativamente con el Desempeño y lo impacta en $4.79 \%$." Estas dimensiones mencionadas en el párrafo anterior explican y permiten "observar" qué la gestión en las (IE) considera cada una de las mismas, como parte de la gestión escolar sin embargo se relaciona negativamente con el desempeño escolar, cuando ésta tiende a ser deficiente.

En las (IE) existen los miembros que mantienen entre sí las relaciones formales que establece la estructura que contempla la gestión escolar. El estudio planteo inicialmente evidenciar si el desempeño escolar se explica por medio de la gestión escolar, para ello se tomó como base las pruebas de lectura y matemática que aplica el Ministerio de Edu- 
cación para estudiantes que están cursando el tercer grado básico, posteriormente se determinó las variables que ocupan en este estudio el desempeño de las (IE). Para ello el modelo contempla variables de proceso como "eficiencia terminal", "resultados en pruebas de lectura y matemática", el modelo de ecuaciones estructurales establece que el desempeño escolar no se explica por la gestión escolar de la (IE) del contexto guatemalteco los resultados son negativos en el desempeño escolar de los estudiantes en las (IE).

La literatura consultada indica que la gestión escolar es un elemento determinante en el desempeño escolar, a medida que se incrementa la descentralización de los procesos de decisión en los sistemas educacionales (Alvariño, et al., 2004), Cassasus (2000) y Rivera (2010) enfatizan la presencia de múltiples procesos en torno a la gestión que abarcan una gran diversidad de actores, recursos y actividades en diferentes órdenes. En ella convergen elementos de carácter institucional, organizacional, político y programático que inciden en la definición de las prácticas educativas en el interior de los centros, y no se reducen a la parte administrativa. Aunado a ello según Pozner (2000), la gestión es una nueva forma de comprender, de organizar y de conducir, tanto al sistema educativo como a la organización escolar; pero esto sólo es así cuando el cálculo estratégico situacional y transformacional se reconoce como uno de sus fundamentos.

Por otro lado la gestión escolar implica además de su organización estructural el entramado de relaciones de interacción entre personas que lo constituye. Como toda institución está compuesta por personas que se relaciona y construyen esquemas de relación entre ellas, comparten ideas, concepciones e intereses no siempre en la misma línea, así la gestión no se reduce a lo burocrático y estructural.

\section{Referencias}

Alvariño, C. y R. Vizcarra (1999), "Gestión para la innovación en educación: desafíos para las escuelas particulares subvencionadas" en Cariola, P y J. Vargas, Educación Particular Subvencionada. CONACEP, Santiago.

Alvariño, C. et al (2000-2004). Gestión escolar: un estado del arte de la literatura. Revista Paideia, 29, pp. 15-43. And managerial applications. New York. Macmilllan.

Arancibia, V. (1992): "Efectividad escolar: un análisis comparado". Estudios Públicos, $\mathrm{N}^{\circ} 47$, pp. 101-125.

Bracho, T. (2009). Innovación en la política educativa. Escuelas de Calidad. Dilemas de las políticas públicas en Latinoamérica. México: flacso.

Canton, Valentona (1997): 1+1+1 no es igual a tres. Ed. Universidad Pedagógica Nacional, México, pp. 13-14.

Casassús, J. (1998). Acerca de la práctica y la teoría de la gestión: Marcos Conceptuales para el análisis de los Cambios en la Gestión de los Sistemas Educativos. Consultado el día 15 de marzo a las 2:50 p.m., de 2015 de la World Wide Web: www.scribd.com/doc/12667410/ gestionbuscadelsujeto.

Casassus Juan (2000) Problemas de la gestión educativa en América Latina (la tensión entre los paradigmas de tipo A y el tipo B).

Coleman J. S. (1969), Equal Educational Opportunity; Harvard University Press, Cambridge Mass. 
Coleman, J. S., Campbell, E. Q., Hobson, C. J., McPartland, J., Mood, A., Weinfield F. D., y York, R. L. (1966). Equality of Educational Opportunity. Washington, DC: Government Printing Office.

Creemers, B. \& J. Scheerens (1994). Development in the educational effectiveness research program. En International Journal of Educational Research, 2 (21), pp. 125-139.

Devellis, R. (2003). Scale development: Theory and applications $\left(2^{\mathrm{a} e d}\right.$.). Newbury Park, CA: Sage.

Espinoza, S. et al (1995): Escuelas de calidad y sus procesos organizacionales: un cambio hacia el mejoramiento de la educación. Tesis para optar al título de Psicólogo. Pontificia Universidad Católica de Chile, Santiago de Chile.

Ezpeleta, J., Furlán A. (1992). La gestión pedagógica de la escuela. Santiago de Chile: UNESCO/OREALC.

Elizondo, A. (Coord.) Bocanegra, N. Gómez, S., González J., Lara, L., Mendieta, Etling, A. (1998). Liderazgo Efectivo, $1^{\text {a }}$. Edición, Trillas, México, p. 10.

Gardner, H. (1995), Inteligencias Múltiples; Paidós, Barcelona.

Gamboa, L., Casas, A. \& Piñeros, J. (2003). La teoría de valor agregado: una aproximación a la calidad de la educación en Colombia. Revista de Economía de la Universidad del Rosario, 6, 95- 116. Disponible en: http://www.urosario.edu. o/ urosario_files/e1/e15b5d6a-dbbb-4b1281d1-b43f057898db.pdf.

Guadamuz, L. (1998).Comentarios. En Namo de Mello G. (1998) Nuevas propuestas para la gestión educativa. México: Biblioteca del Normalista/ SEP.
IIPE (1999), La Formación de Recursos Humanos para la Gestión Educativa en América Latina; IIPE-Buenos Aires.

IIPE-UNESCO (2007). Competencias para la profesionalización de la gestión educativa. Diez módulos destinados a los responsables de los procesos de transformación educativa". Tomado del, Buenos Aires. www.iipe-buenosires.org.ar

Jenks, C. y Bane, M. J. (1972). "Schools and Inequality", en Grass, A. (comp.) [1980]. Sociología de la educación, Madrid: Narcea.

Le Boterf, G Jenks, C. y Bane, M. J. (1972). "Schools and Inequality", en Grass, A. (comp.) [1980]. Sociología de la educación, Madrid: Narcea.

Loera, V. Armando, (2004) Planeación estratégica y política educativa, Documento de trabajo.

Martínez-Arias, R. (2009). Usos, aplicaciones y problemas de los modelos de valor añadido en educación. D.F.: Centro Nacional de Evaluación para la Educación Superior.

Marquiequi, A. (1997). Evaluación de la gestión de un centro educativo en clave de animación. En Revista Educación Hoy, No. 130, enero-junio 1997. pp. 57-76.

Mendoza, M. (2011). Elaboración y validación del cuestionario: "Desempeños profesionales de directivos y profesores en pro de una educación creativa: Evaluación y autoevaluación desde la perspectiva docente". Revista Docencia e Investigación. Nº 21. 2011 ISSN: 1133-9926

MINEDUC (2009) Ministerio de Educación Guatemala. Curriculum Nacional Base (CNB). 
MINEDUC (2005) Ministerio de Educación Chile Curriculum Nacional Base (CNB).

Murnane, R. J. y F. Levy (1996), Teaching the New Basic Skills; Martin Kessler Books The Free Press, New York.

Murillo, F.J. (Coord.) (2007). Investigación iberoamericana sobre eficacia escolar. Colombia: Convenio Andrés Bello.

Murillo.F (2003) Torrecilla. E Garzón .F et al; Investigación sobre Eficacia Escolar OCDE (1992) Schools under Scrutiny; OECD, Paris.

Mourshed, M., Chijioke, C., y Barber, M. (2010). Cómo mejoran los sistemas escolares más mejorados del mundo. Londres: McKinsey \& Company.Nunnally, J., \& Bernstein, I. (1995). Teoría psicométrica. México: McGraw-Hill.

Namo de Mello, G. (1998) Nuevas propuestas para la gestión educativa. México: Biblioteca del Normalista/ SEP.

Navarro, M. (1999). Administración y gestión escolar. Durango, México: SECyD/ CETEB.

OREALC/UNESCO, Oficina Regional para la Educación de América Latina y el Caribe/ Organización de las Naciones Unidas para la Educación, la Ciencia y la Cultura (2010). Factores asociados al logro cognitivo de los estudiantes de América Latina y el Caribe. Santiago de Chile: Oficina Regional para la Educación de América Latina y el Caribe, Organización de las Naciones Unidas para la Educación, la Ciencia y la Cultura, OREALCUNESCO. Disponible en: http://unesdoc.unesco. org/images/0019/001902/190213s.pdf

Pozner de Weinberg, P. (2007). La gestión escolar. En Secretaría de Educación Pública (SEP). Antología de gestión escolar (pp. 35-58). México: SEP.
Pozner de Weinberg, Pilar, (2000). Competencias para la profesionalización de la gestión educativa, capítulo II, iipe, Buenos Aires, 2000.

Perrenoud, Philippe (1998). Construire de compétences dés l'école. Paris. ESF.

Rivera Ferreiro, L. (2010). Elementos conceptuales para el análisis de las prácticas de gestión educativa. En Guerra, M. (coord.). Gestión de la Educación Básica. Referentes, reflexiones y experiencias de investigación (pp. 71-86). México: SEP-UPN

SEP. (2001). ¿Cómo transformar las escuelas? Lecciones desde la gestión escolar y la práctica pedagógica, Subsecretaría de Planeación y Coordinación, Dirección General de Evaluación, Reporte Final, México.

Santos, M. A. (2000). Las relaciones en la escuela: Perspectiva tridimensional. En J. M. Escudero y M. ${ }^{a}$ T. González: Escuelas y profesores ¿Hacia una reconversión de los centros y la función docente? Madrid: Ediciones Pedagógicas. Pp. 115-138.

Servat, B. (1996): "Participación y eficacia en la escuela: estudios empíricos". Boletín de Investigación Educacional № 11, pp. 245-285. Facultad de Educación, Pontifica Universidad Católica de Chile, Santiago de Chile.

Scheerens J. (1992) Effective Schooling. Research, Theory and Practice; Casell, Londres.

Slavin, Robert (1994), "Effective Classrooms, effective schools: a research base for reform in Latin American education". En Puryear, J. y J. J. Brunner (eds.), 1994.

Topete, C. (2001). Desafíos y políticas de formación para la gestión en educación media superior. En Memoria VI Congreso 
Nacional de Investigación Educativa. Universidad de Colima, Manzanillo, 6-10 de noviembre de 2001.

Taylor, P.H. (1962). Childrens Evaluations of the Characteristics of the Good Teacher. British Journal of Educational Psychology, 32(3), 258-266.

Topete, C. (2001). Desafíos y políticas de formación para la gestión en educación media superior. En Memoria VI Congreso Nacional de Investigación Educativa.
Universidad de Colima, Manzanillo, 6-10 de noviembre de 2001.

Tedesco, J.C. (1995). El Nuevo Pacto Educativo. Educación, Competitividad y Ciudadanía en la Sociedad Moderna. Grupo Anaya S.A. Madrid.

Weber, G. (1971). Inner-city children can be taught to read: four successful schools.

Zárate, G (1992): Experiencias educacionales exitosas: un análisis a base de testimonios. Estudios Públicos, № 47, pp. 127-158.

\section{Sobre autora}

\section{Idida Maribel Figueroa Alvarez}

Catedrática de la Universidad de San Carlos de Guatemala, en el Centro Universitario de Petén desde el año 2008, en el 2009 titulación de la Maestría en Educación y Medio Ambiente, posteriormente en el 2015 participo en el doctorado impartido por la Universidad Autónoma de Guadalajara y en el año 2018 me otorgan el grado académico de Dra. en Administración. He participado en el proceso de Investigación de la DIGI-USAC, y actualmente cuento con publicaciones en el Congreso Multidiciplinario de la UJAT, en el libro de la UAG en Villa Hermosa y la Revista Certus Guadalajara.

\section{Sobre coautor}

\section{Amilcar Rolando Corzo Márquez}

Universidad Autónoma de Guadalajara, Campus Tabasco, Profesor Titular del Centro Universitario de Petén, USAC. Licenciado en Pedagogía y Ciencias de la Educación, Facultad de Humanidades, USAC.Licenciado en Arqueología, Centro Universitario de Petén, USAC. Maestría en Medición, Evaluación e Investigación Educativa, (Beca Frank Fairchild, USAID) Universidad del Valle de Guatemala. Doctor en Administración, Universidad Autónoma de Guadalajara. Profesor Titular, CUDEP - USAC.

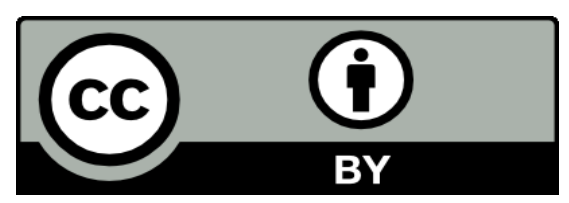

Este texto está protegido por una licencia CreativeCommons 4.0.

Esta licencia permite que otros distribuyan, mezclen, adapten y desarrollen su trabajo, incluso comercialmente, siempre y cuando le den crédito por la creación original. 